\title{
L'utilisation pédagogique de la programmation au primaire : regard sur le développement de compétences transversales
}

\section{CHRONIQUE • Étudiante}

\section{Le contexte}

Dans cette chronique, nous présentons la proposition d'un projet de recherche pour lequel les données nont pas encore été récoltées. Ses fondements originent d'une réflexion sur l'omniprésence du numérique $^{1}$, au moment où le quotidien est agrémenté d'une utilisation fréquente et incontournable du numérique. À travers les pays membres de l'Organisation de coopération et de développement économiques (OCDE), $72 \%$ des élèves mentionnent utiliser l'ordinateur à l'école et $93 \%$ des élèves disent l'utiliser à la maison (OCDE, 2015). Alors que certains s'inquiètent des effets négatifs de cette omniprésence (Siegle, 2017), d'autres en vantent les mérites, lui attribuant la capacité de favoriser l'apprentissage (Freeman et al., 2017). L'adoption d'une position critique et nuancée apparaît alors comme essentielle.

L'utilisation croissante du numérique à l'école est fortement suscitée par les habitudes des apprenants et par l'évolution du contexte dans lequel se déroule l'apprentissage. C'est à cette mutation que le système scolaire doit faire face en modifiant ses fondements, par exemple avec l'apparition d'organisations alternatives de la salle de classe comme la classe inversée (Mazur, Brown et Jacobsen, 2015), ou de dispositifs pédagogiques favorisant l'utilisation d'outils technologiques tels que la réalité virtuelle (Greenwald et al., 2017). Aussi, la programmation ${ }^{2}$ s'inscrit dans cette variété d'outils pertinents pour l'enseignement au $21^{\mathrm{e}}$ siècle, non seulement pour l'attrait qu'elle représente auprès des apprenants (Heflin, Shewmaker et Nguyen, 2017), mais aussi pour ses propriétés potentiellement fortes de développement cognitif (OCDE, 2015). 
Au demeurant, il nous paraît utile de nous pencher sur ce phénomène qui gagne en popularité dans les milieux scolaires. En effet, l'utilisation répandue d'outils de programmation, tels que Scratch Jr. et code.org, démontre l'existence d'un engouement. Bien que l'on puisse attribuer un caractère ludique à ces utilisations, le caractère pédagogique, quant à lui, peut être ignoré, faisant alors des activités de programmation des "passe-temps» peu stimulants. Il convient donc de poser un regard critique sur les pratiques pédagogiques liées aux activités de programmation afin de déterminer si elles contribuent effectivement au développement de compétences.

\section{La programmation et le développement de compétences transversales}

La programmation est une activité d'une grande complexité : le fait d'en comprendre les principes fondamentaux permettrait de changer le rapport qu'entretiennent les personnes avec le numérique, les faisant passer de consommateurs passifs à des consommateurs-créateurs critiques (Romero, 2016). La programmation sollicite la pensée algorithmique ${ }^{3}$, qui mobilise entre autres la résolution de problèmes (Wing, 2006), le raisonnement logique et l'abstraction (The Royal Society, 2012). Dans la mesure où l'informatique est basée sur un principe de traitement de l'information, la programmation est l'outil permettant de diriger ce traitement. Les algorithmes permettent d'y moduler les échanges entre les informations en imposant des conditions ou des itérations (Serafini, 2011).

À l'école primaire, il est possible d'utiliser des dispositifs adaptés de programmation visuelle et concrète. La programmation visuelle, qui utilise une interface graphique plutôt que textuelle, permet non seulement de pallier l'opacité du langage informatique écrit, mais également de contribuer au développement de compétences en résolution de problèmes, notamment en permettant aux élèves de prédire le fonctionnement d'un programme dans un processus itératif et visuel d'essai-erreur (Lai et Yang, 2011). La programmation concrète, quant à elle, consiste à programmer un dispositif physique comme un robot. Cette pratique permet notamment de favoriser le travail collaboratif (Nugent et al., 2009).

$\mathrm{Au}$ vu de ces potentielles retombées, il semble pertinent de mieux comprendre l'utilisation de la programmation au primaire pour le développement de compétences transversales.

Le développement, que l'on évoque ici, s'inscrit dans une perspective à long terme (Liben, 1987), où il y a une progression et une acquisition en profondeur de connaissances (Tardif, 2006). Les compétences transversales, qui sont des savoirs agir allant au-delà du cadre des disciplines (Ministère de l'Éducation, 2006), sont développées non seulement pour être mobilisées en contexte scolaire, mais aussi dans la vie de tous les jours. L'intérêt porté au terme hybride « résolution collaborative de problèmes » (Kamga et al., 2017 ; OCDE, 2017), nous porte à croire qu'il est pertinent de s'intéresser, de façon concomitante, aux compétences de résolution de problèmes et aux compétences de collaboration.

Puisque la programmation pédagogique semble être une occasion propice à la mobilisation et au développement de la résolution de problèmes et de la collaboration, nous choisissons de faire de ces deux compétences le point focal de notre recherche, tels que le définissent nos objectifs de recherche :

[1] Comprendre comment les compétences en résolution de problèmes et en collaboration sont mobilisées lors d'activités de programmation et mesurer leur développement;

[2] Identifier les pratiques de programmation contribuant à la mobilisation de ces compétences. 


\section{CHRONIOUE}

\section{Cadre méthodologique}

Nous avons choisi le devis mixte concomitant triangulé pour structurer notre recherche, notamment parce qu'il permet l'analyse concomitante de données quantitatives et qualitatives, mettant ainsi en lumière des corrélations entre ces deux types de résultats (Fortin et Gagnon, 2016).

Les activités de programmation que nous mettons en place se dérouleront pour une durée indéterminée, c'est-à-dire jusqu'à ce que les élèves aient terminé les activités proposées. Notre intérêt pour le développement des processus cognitifs liés à la résolution de problèmes et des composantes de la collaboration implique qu'il faille constater une évolution. De fait, il est nécessaire de collecter des données avant, pendant et après la série d'activités auprès des 70 élèves de troisième et de quatrième année du primaire composant notre échantillon. Les instruments utilisés sont le questionnaire, l'observation et les entrevues : ils fonctionnent de façon complémentaire, puisque les données de chacun permettent de préciser ou de justifier les données des autres. La composante qualitative, liée à la collecte des points de vue des participants à l'étude, aura pour effet principal de permettre la triangulation des données issues de la composante quantitative, rattachée à la mesure de la compétence en résolution de problèmes et en collaboration.

Avec cette recherche, nous espérons contribuer à la réflexion sur le potentiel pédagogique de la programmation à l'école primaire. Nous souhaitons que notre recherche permette aux enseignants de construire des activités qui ciblent et développent les compétences en résolution de problèmes et en collaboration.

\section{Notes}

1 Nous appuyons notre compréhension du terme numérique sur les propos de Vitali-Rosati (2014).

2 La programmation est l'action d'écrire, à l'aide d'un langage informatique, une série d'actions qui sont interprétées puis exécutées par un ordinateur (Blackwell, 2002).

3 Il s'agit d'une " procédure systématique permettant de résoudre une classe de problèmes. À partir d'une entrée (représentant une instance du problème), un algorithme suit un ensemble déterminé de règles et, en un nombre fini d'étapes, produit une sortie (représentant une réponse à l'instance donnée) » (Modeste, 2012, p. 468)

\section{Références}

Fortin, F. et Gagnon, J. (2016). Fondements et étapes du processus de recherche : méthodes quantitatives et qualitatives. ( $3^{\mathrm{e}}$ éd.). Montréal, Canada: Chenelière éducation.

Freeman, A., A.-Becker, S., Cummins, M., Davis, A. et H.-Giesinger, C. (2017). NMC/CoSN Horizon Report: 2017 K-12 Edition. Austin, États-Unis: The New Media Consortium.

Greenwald, S., Kulik, A., Kunert, A., Beck, S., Frohlich, B., Cobb, S., .. Cook, C. (2017, 18-22 juin). Technology and applications for collaborative learning in virtual reality. Communication présentée à 12th International Conference on Computer Supported Collaborative Learning (CSCL), Pensylvanie, États-Unis.

Heflin, H., Shewmaker, J. et Nguyen, J. (2017). Impact of mobile technology on student attitudes, engagement, and learning. Computers E' Education, 107(1), 91-99.

Kamga, R., Romero, M., Komis, V. et Misirli, A. (2017, 18-19 mai). Identification des difficultés des futur(e)s enseignant(e)s du primaire et du préscolaire en lien avec la compétence de résolution collaborative de problèmes (RCP). Communication présentée au 5e Sommet du iPad et du numérique en éducation, Montréal, Canada. 
Lai, A.-F. et Yang, S.-M. (2011, 16-18 septembre). The learning effect of visualized programming learning on 6 th graders' problem solving and logical reasoning abilities. Communication présentée à International Conference on Electrical and Control Engineering (ICECE), Yichang, Chine.

Liben, L. S. (1987). Approaches to Development and Learning: Conflict and Congruence. Dans L. S. Liben (dir.), Development and learning : conflict or congruence? (p. 225-235). New Jersey, États-Unis: L. Erlbaum Associates.

Mazur, A. D., Brown, B. et Jacobsen, M. (2015). Conception d'apprentissage à l'aide de l'instruction en classe inversée. La Revue canadienne de l'apprentissage et de la technologie, 41(2). doi: 10.21432/t2pg7p

Ministère de l'Éducation, du Loisir et du Sport. (2006). Programme de formation de l'école québécoise - Enseignement primaire. Québec, Canada: Gouvernement du Québec.

Modeste, S. (2012, février). La pensée algorithmique: apports d'un point de vue extérieur aux mathématiques. Communication présentée au Colloque Espace Mathématique Francophone, Genève, Suisse.

Nugent, G., Barker, B., Grandgenett, N. et Adamchuk, V. (2009, 18-21 octobre). The use of digital manipulatives in k-12. robotics, GPS/GIS and programming. Communication présentée à la 39th ASEE/IEEE Frontiers in Education Conference, Texas, États-Unis. doi: 10.1109/FIE.2009.5350828

OCDE. (2015). Students, Computers and Learning: Making the Connection PISA. OECD Publishing.

OCDE. (2017). PISA 2015 Results, Collaborative Problem Solving. OECD Publishing.

Romero, M. (2016). Faut-il enseigner l'art du code à nos jeunes? Dans G. Murchison (dir.), L'actuel: Radio-Canada.

Schalow, F. (2017). Technology and the Rise of the Artifice. Dans F. Schalow (dir.), Toward a Phenomenology of Addiction: Embodiment, Technology, Transcendence (p. 89-113). Cham: Springer International Publishing.

Serafini, G. (2011, octobre). Teaching programming at primary schools: visions, experiences, and long-term research prospects. Communication présentée à International Conference on Informatics in Schools: Situation, Evolution, and Perspectives, Bratislava, Slovaquie.

Tardif, J. (2006). Lévaluation des compétences : documenter le parcours de développement. Montréal, Canada: Chenelière Éducation.

The Royal Society. (2012). Shut down or restart? The way forward for computing in UK schools. Londres, Angleterre: The Royal Society, Education Section.

Wing, J. M. (2006). Computational thinking. Communications of the ACM, 49(3), 33-35.

\section{Pour citer cet article}

Parent, S. (2017). L'utilisation pédagogique de la programmation au primaire : regard sur le développement de compétences transversales. Formation et profession, 25(3), 130-133. http://dx.doi.org/10.18162/fp.2017.a137 